\title{
Perfil del administrador: construcción a partir de la experiencia Tuning-Colombia ${ }^{1}$
}

DOI: http://dx.doi.org/10.17981/econcuc.37.2.2016.02

\author{
Javier Moreno Juvinao ${ }^{2}$ \\ Jaime Castrillón Cifuentes ${ }^{3}$ \\ John Sánchez Otero ${ }^{4}$ \\ Ana Virginia Moreno Charris ${ }^{5}$
}

\section{Resumen}

Las tendencias orientadas hacia la calidad de la educación superior se han fundamentado en la renovación de metodologías educativas, que trascienden de modelos centrados en la enseñanza hacia el aprendizaje basado en el desarrollo de competencias. En función de ello, se concibe el objetivo de determinar el perfil del administrador de empresas egresado de la Universidad de la Costa-Colombia, para contrastarlo con las competencias definidas en el proyecto Tuning-Colombia. Se establece una investigación descriptiva. Las unidades muestrales fueron localizadas en la Universidad de la Costa, como contexto organizacional donde se configura el componente empírico de la investigación. El uso del método cuantitativo, identificó los resultados que determinan las bases del desarrollo de competencias, donde se prevé la compatibilidad, competitividad y el análisis de las demandas del sector, considerado como componentes integradores para la adaptación de diseños curriculares. Se concluye, que concebir la formación en competencias consolida la formación integral de profesionales capaz de transformar contextos sociales y organizacionales dinámicos y complejos, por lo que se recomienda afianzar la calidad del proceso educativo superior como referencia que confluye con los avances impulsados desde un entorno global.

\footnotetext{
${ }^{1}$ Artículo científico derivado de la investigación "Competencias de los administradores de la Universidad de la Costa - CUC a la luz del Proyecto Tuning Colombia”, financiada por la Universidad de la Costa CUC.

${ }^{2}$ Candidato a Magister en Administración de empresas. Universidad del Norte. Especialista en Finanzas y Sistemas. Especialista en Estudios Pedagógicos. Universidad de la Costa. Especialista en Derecho del Medio Ambiente. Universidad Externado de Colombia. Investigador adscrito al Grupo de Investigación Administración Social. Decano de la Facultad de Ciencias Económicas de la Universidad de la Costa. Autor de correspondencia, jjmorenoj@cuc.edu.co
}

${ }^{3}$ MBA. Universidad EAFIT Medellín. Licenciado en Educación. Universidad de San Buenaventura Medellín. Profesor adscrito a la Escuela de Negocios Universidad del Norte. jcastril@uninorte.edu.co

${ }^{4}$ Candidato a Magister en gestión de la Innovación. Universidad Tecnológica de Bolívar. Ingeniero Mecánico. Universidad del Norte. Jefe de la Unidad de I+D+i de la Universidad de la Costa CUC. Jsanchez17@cuc.edu.co; jesf7@hotmail.com

${ }^{5}$ Maestrante en Administración. Universidad de la Costa CUC. Profesional en Relaciones Internacionales. Universidad del Norte. Profesora e Investigadora adscrita al grupo Administración Social de la Universidad de la Costa. amoreno14@cuc.edu.co 
Palabras clave: perfil del administrador; competencias genéricas; competencias específicas; Proyecto Tuning; Universidad de la Costa-Colombia.

\title{
Profile of an administrator: construction since the tuning colombia experience
}

\begin{abstract}
Trends orientated to higher education quality have been rooted on the renewal of educational methodologies, which trascend from teaching-focused models to the learning based on the competencies development. Depending on it, it is conceive the goal of determining the profile of the business administrators graduated from Universidad de la Costa-Colombia, to contrast it with the competencies defined in the Tuning-Colombia project. It is established a descriptive research. The sample units were located in University of the Coast, as an organizational context where the empirical component of the research is configured. The use of the quantitative method, identified the results that determine the bases of the development of competences, where the compatibility, competitiveness and the analysis of the sector demands are envisaged, considered as integrating components to the adaptation of the curricular designs. It is concluded that conceiving the training in competencies consolidates professionals integral training capable of transforming dynamic and complex social and organizational contexts, which is why it is recommended secure the higher education process quality as a reference that comes together with the advances promoted from a global environment.
\end{abstract}

Keywords: administrator profile; generic competencies; specific competencies; Tuning Project; Universidad de la Costa-Colombia.

\section{Introducción}

Los avances y resultados representados a través de la formación de profesionales que egresan de las instituciones de educación superior responden al desarrollo alcanzado por las funciones básicas centradas en la docencia, investigación y extensión que se promueven en el sector. Sin embargo, en la última década importantes iniciativas han 
sido orientadas por organismos internacionales como la Organización de las Naciones Unidas para la Educación, la Ciencia y la Cultura (UNESCO). Las tendencias educativas que se impulsan y promueven inciden en los procesos formativos que han sido contextualizados en diferentes países; su impacto se proyecta en el diseño de estrategias que redundan en el proceso de enseñanza-aprendizaje que, al ser concebidos de forma integral, contribuyen asertivamente con el desarrollo socioeducativo de los países.

En el marco de los avances alcanzados se menciona que desde el año 2000, el continente europeo inicia un proyecto denominado Tuning Educational Structures in Europe, cuyo objetivo central consistió en crear un área de educación superior integrada en el trasfondo de un área social y económica. Esta iniciativa se desarrolló en el contexto de la reflexión sobre la educación superior que se ha impuesto como consecuencia del acelerado ritmo de cambio de la sociedad y de la necesidad de establecer factores de compatibilidad, comparabilidad y competitividad de la educación superior que permitieran a los estudiantes tener información fiable y objetiva sobre la oferta de programas educativos, y, a los empresarios, obtener información confiable sobre lo que ellos significan en la práctica de un título determinado (Bravo, 2007).
El proyecto Tuning Europa alcanzó una aceptación significativa, debido a que la idea central no fue establecer títulos ni planes de estudios unificados, prescriptivos o definitivos (Universidad de Deusto Universidad de Groningen, 2007), sino que el interés consistió en establecer puntos comunes de referencia, estimular la convergencia y la comprensión. Lo anterior ha facilitado el intercambio de estudiantes; la búsqueda de empleos en cualquier país de la Unión Europea; la participación activa de los involucrados: universidades, Estado, estudiantes, empresarios; alto nivel de consenso con respecto a la educación; y se ha dejado espacio para la diversidad, la libertad y la autonomía.

Esta experiencia fue transferida a Latinoamérica en el marco del proyecto Tuning América Latina 2004 - 2006, liderado por latinoamericanos con el acompañamiento de expertos europeos. Los resultados del proyecto Tuning América Latina remiten implícitamente a un marco de reflexión y crítica en torno a la educación superior, producto de una multi-referencialidad tanto pedagógica como disciplinaria.

Desde esta perspectiva, han sido considerados factores determinantes para impulsar el proyecto desde el contexto colombiano, cuyo fin era el de contrastar y comparar los diferentes aspectos de la 
diversidad de los países latinoamericanos frente a los programas nacionales (Aguilar, 2011). En función de ello, el análisis sobre los enfoques del modelo basado en competencias, así como el impacto sobre la tendencia en el sector educativo superior y su expansión hacia el contexto latinoamericano, han sido considerados como parte de las premisas para el desarrollo del objetivo centrado en identificar el perfil de competencias del administrador de empresas de la Universidad de la Costa, Colombia, para contrastarlas con las competencias establecidas en el proyecto $\mathrm{Tu}$ ning-Colombia.

Para su argumentación, se fundamentan los principales aspectos teóricos relacionados con el proyecto Tuning y sus bases centradas en el desarrollo de competencias, así como también se caracteriza el perfil del administrador según la concepción de procesos formativos integrales basados en la enseñanza de la administración según tendencias promovidas desde un orden global y local. Para los efectos de su contextualización, se considera la definición de competencias genéricas y específicas según el grupo de interés (estudiantes, egresados, académicos y empresarios), las cuales se convirtieron en el fundamento para la construcción de un perfil del administrador de empresas de la Universidad de la Costa que esté alineado con el currí- culo para responder a la convergencia de programas de administración de empresas compatibles para afianzar procesos de intercambios, prácticas, homologaciones, empleos y movilidad en general.

\section{Proyecto Tuning: Alcance $y$ tendencia desde la realidad de la educación superior}

Una de las principales tendencias que ha generado importantes transformaciones en la educación superior ha sido la experiencia promovida a través del Proyecto Tuning, cuyo propósito central desde el contexto Latinoamericano y su expansión hacia el Tuning Colombia, consistió en establecer puntos de referencia, respetando la diversidad de la educación superior nacional y la autonomía universitaria. Con esta iniciativa se ha buscado: "contribuir con el desarrollo de titulaciones fácilmente comparables y comprensibles, desde los objetivos que la titulación determine, hasta los perfiles buscados para los egresados, ofreciendo elementos que posibiliten la movilidad de los poseedores de títulos profesionales en Colombia" (Beneitone, Esquetini, González, Maletá, Siufi y Wagenaar, 2007, p.15).

En este orden de ideas, se expone según la UNESCO (2009) la importancia de re- 
flejar los objetivos generales de la educación superior $\mathrm{y}$, por ende, hacer énfasis en la formación a lo largo de la vida impulsando como meta básica el cultivar el pensamiento crítico e independiente, así como también promover la innovación y la diversidad multicultural para afrontar retos desde una perspectiva crítica y con impacto social.

Entre las propuestas desarrolladas alrededor del proyecto Tuning en los diferentes países de Latinoamérica, Colombia no ha sido la excepción al orientar sus esfuerzos en definir competencias que sustenten los programas académicos, no sólo desde un contexto local, sino desde una mirada global e internacional. A través de este proceso se busca responder a la movilidad de estudiantes y profesionales, además de las necesidades de empresarios ubicados en diferentes espacios geográficos. No se puede dejar de destacar que el objetivo estuvo centrado en establecer un escenario para la identificación e intercambio de la información y el mejoramiento de la colaboración entre las instituciones de educación superior para el desarrollo de la calidad, efectividad y transparencia (Beneitone et al, 2007).

En líneas generales, la definición de competencias articuladas con los diseños curriculares necesariamente han de respon- der con el desarrollo de potencialidades en el individuo para interactuar en diferentes contextos, según se indican: las competencias a desarrollar desde el ambiente de las empresas y las que se afianzan a través de la formación formal desde las instituciones educativas, donde la interacción entre la práctica y fundamentación teórica hace parte de la estrategia formativa.

\section{Bases para el diseño curricular en el marco del desarrollo de competencias}

Como aspecto fundamental para el desarrollo y transformación hacia la calidad educativa promovida por las instituciones de educación superior, la reestructuración del diseño curricular constituye un componente estratégico para consolidar las fases de la formación integral del individuo. En tal sentido, la concepción de un nuevo diseño curricular fundamentado en el desarrollo de competencias es parte de los retos por afianzar, sin dejar de destacar los avances alcanzados en la actualidad, sobre todo en el contexto latinoamericano.

Como aspecto influyente para las bases del diseño curricular se menciona uno de los principales componentes vinculantes, como lo es la articulación entre la educación superior y el sector productivo, que 
en determinados casos genera una débil inclusión que disocia el fin último; es oportuno mencionar la participación de este sector con sentido de pertinencia y los aportes que ha generado en función de los estándares exigidos para la empleabilidad de los egresados y la capacidad de respuesta ante las necesidades de los empresarios.

En función de lo descrito, se requiere mejorar e incluir en los programas, como sea posible, las siguientes consideraciones: (i) módulos sobre competencias generales demandadas por las empresas de todos los países (análisis de problemas, organización del tiempo, correcta escritura y trabajo en equipo); (ii) prácticas empresariales como una parte integral del programa; (iii) evaluación de la evolución de los estudiantes que incluya la valoración de la empresa en la que hayan realizado las prácticas (Organización para la Cooperación y el Desarrollo Económico y Banco Mundial, 2012).

Otro aspecto importante con relación a la articulación educativa responde a la internacionalización. En contextos como el colombiano, según la Asociación Colombiana de Facultades de Administración - ASCOLFA (2010), las instituciones de educación superior han construido las competencias de sus programas de administración, quizás mirando sólo su entorno y dejando de lado los escenarios internacionales. Según expone Valero (2011), el actual proceso de globalización está caracterizado, entre otras cosas, por la creciente movilidad de estudiantes y profesionales, que requiere información fiable y objetiva sobre la oferta de programas educativos, además de las necesidades de empresarios ubicados en diferentes espacios geográficos".

Es claro que en este contexto se generan necesidades de movilidad de estudiantes y egresados tanto en el ámbito nacional como internacional, a las cuales los programas deben responder; sin embargo, estos no se han ajustado a la globalización, que sin duda alguna, impacta los procesos de educación. Es por ello que el proyecto Tuning-Europa fue adaptado en diferentes países de América Latina, desarrollándose, en el caso de Colombia, un proyecto entre varias universidades locales en el marco del estudio del área temática de administración de empresas, lo que permitió analizar las competencias cruciales relacionadas con el conocimiento concreto en el área. Esas competencias confieren identidad y consistencia a los programas de administración de empresas, y por ende, impactan en el proceso formativo del profesional con competencias centradas en la competitividad y el emprendimiento como factor básico de diferenciación. 


\section{Metodología}

El enfoque metodológico se fundamentó en una investigación descriptiva y el uso del método cuantitativo. Las unidades muestrales fueron localizadas en la Universidad de la Costa, como contexto organizacional donde se configura el componente empírico de la investigación. La categorización de la muestra seleccionada estuvo conformada por académicos, estudiantes, empresarios y egresados, denominados grupo de consul$t a$, la cual se representó a través de 1.485 individuos categorizados de acuerdo con el grupo de consulta al que pertenecen.

Para la selección de la muestra se estableció el método aleatorio estratificado, usando un criterio de fijación proporcional, lo cual permitió considerar una muestra representativa de 542 personas (tabla 2), con una confiabilidad del 96 por ciento, discriminada por estrato, así:

$$
n=\frac{N}{1+N e^{2}}=\frac{1485}{1+1485(0.0342)^{2}}=542
$$

La técnica seleccionada fue la encuesta, la cual se aplicó a través de un cuestionario con el fin de obtener información susceptible de análisis sobre las competencias genéricas y específicas de los administradores de la Universidad de la Costa, las cuales fueron calificadas de acuerdo con las siguiente
Tabla 1

Grupos de consulta.

\begin{tabular}{lccc}
\hline \multicolumn{1}{c}{ Estrato } & Población & $\begin{array}{r}\text { Peso de } \\
\text { estrato }\end{array}$ & Muestra \\
\hline Estudiantes & 1225 & 0,825 & 448 \\
Empresarios & 70 & 0,047 & 25 \\
Egresados & 130 & 0,088 & 48 \\
Académicos & 60 & 0,040 & 21 \\
Total & 1485 & 1 & 542 \\
\hline
\end{tabular}

Fuente: elaboración propia.

escala: $1=$ nada; $2=$ poco; $3=$ bastante; 4 $=$ mucho. Para el procesamiento de la información obtenida se utilizó la herramienta estadística SPSS versión 20.0.

Para el proceso de evaluación de competencias, se consideró la identificación que realizó Colombia desde la experiencia Tuning, desarrollada por ASCOLFA (2010), mediante la cual se seleccionó un grupo de 27 competencias genéricas y 20 competencias específicas para el administrador de empresas. No obstante, en un estudio más actualizado, realizado por la Universidad de Deusto (2014), se definió que las 27 competencias estaban vigentes y que se debía incorporar una competencia adicional considerada con alta importancia para los programas de administración identificada como la capacidad de emprendimiento e innovación con un alcance amplio, no sólo para emprender nuevos negocios sino 
también proyectos individuales, sociales y gubernamentales, con el componente de innovación para la generación de valor agre- gado. Dado lo anterior, ésta se incorporó en la lista de competencias genéricas, relacionadas en la tabla 2 .

Tabla 2

Registro de competencias genéricas.

\section{Descripción de la competencia}

1. Capacidad de abstracción, análisis y síntesis

2. Capacidad de aplicar los conocimientos en la práctica

3. Capacidad para organizar y planificar el tiempo

4. Conocimientos sobre el área de estudio y la profesión

5. Responsabilidad social y compromiso ciudadano

6. Capacidad de comunicación oral y escrita

7. Capacidad de comunicación en un segundo idioma

8. Habilidades en el uso de las tecnologías de la información y de la comunicación

9. Capacidad de investigación

10. Capacidad de aprender y actualizarse permanentemente

11. Habilidades para buscar, procesar y analizar información procedente de fuentes diversas

12. Capacidad crítica y autocrítica

13. Capacidad para actuar en nuevas situaciones

14. Capacidad creativa

20. Compromiso con la preservación del medio ambiente

21. Compromiso con su medio socio-cultural

22 . Valoración y respeto por la diversidad y multiculturalidad

23. Habilidad para trabajar en contextos internacionales

24. Habilidad para trabajar en forma autónoma

25. Capacidad para formular y gestionar proyectos

26. Compromiso ético

27. Compromiso con la calidad

28. Emprendimiento e innovación (adicional)

Fuente: elaboración propia. Adaptado de ASCOLFA (2010). 
Tabla 3

Lista de competencias especificas.

Descripción de la competencia

1. Desarrollar un planeamiento estratégico, táctico y operativo

2. Identificar y administrar los riesgos de negocios de las organizaciones

3. Identificar y optimizar los procesos de negocio de las organizaciones

4. Administrar un sistema logístico integral

5. Desarrollar, implementar y gestionar sistemas de control administrativo

6. Identificar las interrelaciones funcionales de la organización

7. Evaluar el marco jurídico aplicado a la gestión empresarial

8. Elaborar, evaluar y administrar proyectos empresariales en diferentes tipos de organizaciones

9. Interpretar la información contable y financiera para la toma de decisiones gerenciales

10. Usar la información de costos para el planeamiento, el control y la toma de decisiones

11. Tomar decisiones de inversión, financiamiento y gestión de recursos financieros en la empresa

12. Ejercer el liderazgo para el logro y consecución de metas en la organización

13. Administrar y desarrollar el talento humano en la organización

14. Identificar aspectos éticos y culturales de impacto recíproco entre la organización y el entorno social

15. Mejorar e innovar los procesos administrativos

16. Detectar oportunidades para emprender nuevos negocios y/o desarrollar nuevos productos

17. Utilizar las tecnologías de información y comunicación en la gestión

18. Administrar la infraestructura tecnológica de una empresa

19. Formular y optimizar sistemas de información para la gestión

20. Formular planes de marketing

21. Administrar los recursos humanos, físicos, financieros y otros de la organización (adicional)

Fuente: Adaptado de ASCOLFA (2010).

Asimismo, en el estudio antes referido se concluyó la falta de incorporación de una nueva competencia específica: administrar los recursos humanos, físicos, financieros y otros de la organización, en donde la palabra «otros» contempla la tecnología, la 
información, el conocimiento, etc. Por tal razón, ésta también se adicionó en el listado de competencias específicas, tal como se muestra en la tabla 3.

Con relación a la valoración de las competencias, se consultaron tres variables que determinan:

1. Grado de importancia: relevancia de la competencia, en su opinión, para el trabajo de su profesión.

2. Grado de desarrollo: logro o alcance de esa competencia como resultado del proceso de formación.

3. Ranking de importancia: orden de importancia de las competencias (genéricas y específicas), que según la apreciación de los grupos de consulta, se definieron en las cinco más importantes en función de la categoría que otorgaron los encuestados.

Con la información obtenida, se realizó un análisis por grupo de consulta con relación a los factores de importancia y realización a través del cual se determinaron las cinco competencias más importantes por grupo. Este proceso permitió, tanto el análisis de competencias, como el de los grupos de consulta relacionados con los resultados obtenidos en Tuning Colombia.

\section{Resultados}

La presentación de los resultados se fundamenta de acuerdo con la obtención de información producto de la aplicación del cuestionario. Para los efectos, se analizaron los datos obtenidos en función de las diferentes unidades muestrales seleccionadas, considerando la relación sobre las competencias genéricas y específicas, así como su importancia y grado de desarrollo. El referido proceso consistió en una prueba de normalidad de los datos, por lo que se inició con las estadísticas de centramiento y dispersión de cada una de las variables medidas y su estadístico de contraste para verificar la normalidad.

En la tabla 4, se registran los resultados de la prueba de normalidad, allí se observa que la mayoría de las variables estudiadas mantienen los valores de la sigma asintótica (P-valor) por encima del nivel de significancia fijado por defecto que es 0.05 (equivalente a un grado de confianza del 95\%). Esto no es más que la probabilidad de rechazar la hipótesis nula cuando realmente es verdadera, es decir, la probabilidad de cometer un error de tipo I o uno de los casos donde se puede optar por una decisión incorrecta. El cumplimiento del presente supuesto abre el camino para los contrastes de medias aritméticas de las competencias genéricas y específicas del programa de Ad- 
Tabla 4

Pruebas de normalidad univariada.

\begin{tabular}{|c|c|c|c|c|c|}
\hline \multirow[b]{2}{*}{ Variable } & \multicolumn{3}{|c|}{ Parámetros normales } & \multirow{2}{*}{$\begin{array}{c}\mathrm{Z} \text { de } \\
\text { Kolmogorov- } \\
\text { Smirnov }\end{array}$} & \multirow{2}{*}{$\begin{array}{c}\text { Sig. } \\
\text { Asintótica } \\
\text { (bilateral) }\end{array}$} \\
\hline & $\mathrm{N}$ & Media & $\begin{array}{l}\text { Desviación } \\
\text { típica }\end{array}$ & & \\
\hline Importancia de las competencias específicas en estudiantes & 21 & 3,5868 & 0,0716 & 0,6162 & 0,8421 \\
\hline $\begin{array}{l}\text { Nivel de realización de las competencias específicas en } \\
\text { estudiantes }\end{array}$ & 21 & 3,1263 & 0,0936 & 0,5771 & 0,8930 \\
\hline Importancia de las competencias genéricas en estudiantes & 28 & 3,5850 & 0,1041 & 0,8549 & 0,4579 \\
\hline $\begin{array}{l}\text { Nivel de realización de las competencias genéricas en } \\
\text { estudiantes }\end{array}$ & 28 & 3,1749 & 0,2026 & 1,4106 & 0,0574 \\
\hline Importancia de las competencias específicas en académicos & 21 & 3,6373 & 0,1283 & 0,9033 & 0,3882 \\
\hline $\begin{array}{l}\text { Nivel de realización de las competencias específicas en } \\
\text { académicos }\end{array}$ & 21 & 3,3221 & 0,1936 & 1,0534 & 0,2171 \\
\hline Importancia de las competencias genéricas en académicos & 28 & 3,7174 & 0,1394 & 0,8249 & 0,5043 \\
\hline Nivel de realización de las competencias genéricas en académicos & 28 & 3,2384 & 0,1890 & 0,8159 & 0,5184 \\
\hline Importancia de las competencias específicas en graduados & 21 & 3,5853 & 0,1369 & 1,1156 & 0,1659 \\
\hline $\begin{array}{l}\text { Nivel de realización de las competencias específicas en } \\
\text { graduados }\end{array}$ & 21 & 2,9600 & 0,1724 & 0,4412 & 0,9900 \\
\hline Importancia de las competencias genéricas en graduados & 28 & 3,6044 & 0,1534 & 0,6959 & 0,7180 \\
\hline Nivel de realización de las competencias genéricas en graduados & 28 & 2,9497 & 0,3092 & 0,9657 & 0,3086 \\
\hline Importancia de las competencias específicas en administrativos & 21 & 3,5445 & 0,1981 & 0,7723 & 0,5898 \\
\hline $\begin{array}{l}\text { Nivel de realización de las competencias específicas en } \\
\text { administrativos }\end{array}$ & 21 & 3,3437 & 0,2246 & 0,4786 & 0,9760 \\
\hline Importancia de las competencias genéricas en administrativos & 28 & 3,5419 & 0,1675 & 0,8595 & 0,4511 \\
\hline $\begin{array}{l}\text { Nivel de realización de las competencias genéricas en } \\
\text { administrativos }\end{array}$ & 28 & 3,4332 & 0,1921 & 0,6033 & 0,8599 \\
\hline Importancia de las competencias específicas en el país & 20 & 3,5910 & 0,1163 & 0,5740 & 0,8967 \\
\hline Nivel de realización de las competencias específicas en el país & 20 & 2,9865 & 0,1190 & 1,0206 & 0,2486 \\
\hline Importancia de las competencias genéricas en el país & 27 & 3,5700 & 0,1494 & 0,6171 & 0,8409 \\
\hline Nivel de realización de las competencias genéricas en el país & 27 & 2,9026 & 0,3189 & 0,7346 & 0,6531 \\
\hline
\end{tabular}

Fuente: elaboración propia. 
ministración de Empresas de la Universidad de la Costa con respecto a lo contenido en el nivel de país. De no haberse cumplido este supuesto, sería necesario mirar otras técnicas en la estadística no paramétrica que toman como referencia estadísticos como la mediana. Este fenómeno se debe a que cuando la normalidad de los datos no se cumple es porque los datos están muy dispersos con respecto a la media aritmética o al evento asociado, o a que existen datos atípicos.

En función de la referida prueba de normalidad univariada y el gráfico de dis- persión que se muestra en la figura 1 , se puede afirmar que la tendencia lineal de los datos es muy cercana a la de una función de grado uno con pendiente cercana a cero. Es decir, que en la recurrencia de los eventos y en la ley de los grandes números se espera un grado de confianza del $95 \%$ y que el coeficiente de la variable independiente esté entre $-1,86 \times 10-5$ y 0,00957 . Por tal razón, se considera no rechazar la hipótesis nula donde dice que el coeficiente de la variable independiente estadísticamente es cero, con lo que se reafirma la tendencia lineal de las variaciones con pendiente tendiente a cero.

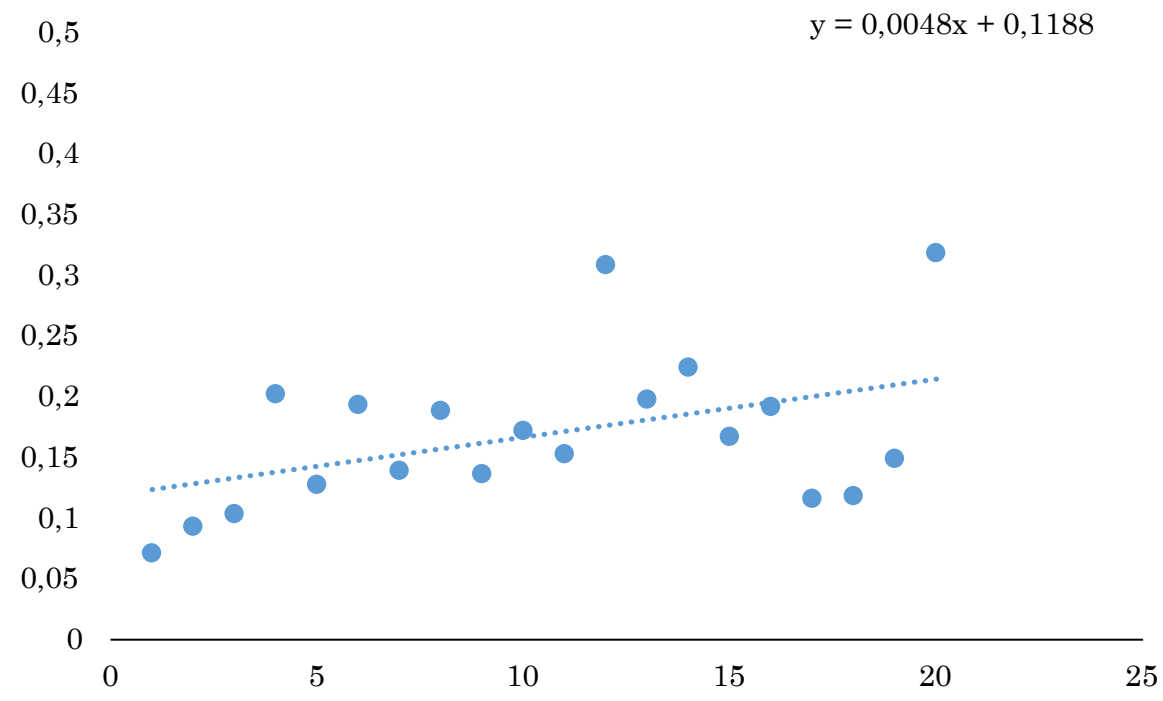

Figura 1. Gráfico de dispersión por cada variable estudiada.

Fuente: elaboración propia. 
Esta prueba de normalidad permitió analizar los datos a través de la estadística paramétrica. Se realizó un análisis orientado a contrastar el grado de importancia y desarrollo de las competencias genéricas y específicas de los diferentes actores estudiados y la construcción de un ranking a partir del contraste en función del nivel de importancia para los grupos de análisis.
El otro análisis realizado se enfocó en contrastar la percepción de los grupos de interés con relación a la percepción nacional en Colombia, por lo que se hizo necesario establecer los contrastes estadísticos para muestras relacionadas tomando como referencia la media aritmética y también unas correlaciones bivariadas con el coeficiente de Sperman.

Tabla 5

Resultados del análisis para las competencias genéricas.

\begin{tabular}{|c|c|c|c|c|}
\hline $\begin{array}{l}\text { Ranking de } \\
\text { competencias } \\
\text { genéricas: } \\
\text { importancia }\end{array}$ & Estudiantes & Académicos & Graduados & Empresarios \\
\hline 1 & $\begin{array}{l}\text { 17. Capacidad de } \\
\text { trabajo en equipo. }\end{array}$ & $\begin{array}{l}\text { 26. Compromiso } \\
\text { ético. }\end{array}$ & $\begin{array}{l}\text { 28. Emprendimiento e } \\
\text { innovación }\end{array}$ & $\begin{array}{l}\text { 17. Capacidad de } \\
\text { trabajo en equipo }\end{array}$ \\
\hline 2 & $\begin{array}{l}\text { 27. Compromiso } \\
\text { con la calidad }\end{array}$ & $\begin{array}{l}\text { 27. Compromiso con } \\
\text { la calidad }\end{array}$ & 26. Compromiso ético & $\begin{array}{c}\text { 2. Capacidad } \\
\text { de aplicar los } \\
\text { conocimientos en la } \\
\text { práctica }\end{array}$ \\
\hline 3 & $\begin{array}{l}\text { 16. Capacidad } \\
\text { para tomar } \\
\text { decisiones }\end{array}$ & $\begin{array}{l}\text { 1. Capacidad de } \\
\text { abstracción, análisis } \\
\text { y síntesis }\end{array}$ & $\begin{array}{l}\text { 27. Compromiso con la } \\
\text { calidad }\end{array}$ & $\begin{array}{l}\text { 15. Capacidad para } \\
\text { identificar, plantear } \\
\text { y resolver problemas }\end{array}$ \\
\hline 4 & $\begin{array}{l}\text { 26. Compromiso } \\
\text { ético }\end{array}$ & $\begin{array}{c}\text { 2. Capacidad } \\
\text { de aplicar los } \\
\text { conocimientos en la } \\
\text { práctica }\end{array}$ & $\begin{array}{l}\text { 10. Capacidad } \\
\text { de aprender y } \\
\text { actualizarse } \\
\text { permanentemente. }\end{array}$ & $\begin{array}{l}\text { 26. Compromiso } \\
\text { ético }\end{array}$ \\
\hline 5 & $\begin{array}{l}\text { 4. Conocimientos } \\
\text { sobre el área } \\
\text { de estudio y la } \\
\text { profesión }\end{array}$ & $\begin{array}{l}\text { 16. Capacidad para } \\
\text { tomar decisiones }\end{array}$ & $\begin{array}{l}\text { 17. Capacidad de } \\
\text { trabajo en equipo }\end{array}$ & $\begin{array}{c}\text { 6. Capacidad de } \\
\text { comunicación oral y } \\
\text { escrita }\end{array}$ \\
\hline
\end{tabular}




\begin{tabular}{|c|c|c|c|c|}
\hline $\begin{array}{l}\text { Ranking de } \\
\text { competencias } \\
\text { genéricas: } \\
\text { grado de } \\
\text { desarrollo }\end{array}$ & Estudiantes & Académicos & Graduados & Empresarios \\
\hline 1 & $\begin{array}{l}\text { 17. Capacidad de } \\
\text { trabajo en equipo }\end{array}$ & $\begin{array}{l}\text { 4. Conocimientos } \\
\text { sobre el área de } \\
\text { estudio y la profesión }\end{array}$ & 26. Compromiso ético & $\begin{array}{l}\text { 17. Capacidad de } \\
\text { trabajo en equipo }\end{array}$ \\
\hline 2 & $\begin{array}{l}\text { 4. Conocimientos } \\
\text { sobre el área } \\
\text { de estudio y la } \\
\text { profesión }\end{array}$ & 26. Compromiso ético & $\begin{array}{l}\text { 27. Compromiso con la } \\
\text { calidad }\end{array}$ & $\begin{array}{l}\text { 18. Habilidades } \\
\text { interpersonales }\end{array}$ \\
\hline 3 & $\begin{array}{l}\text { 26. Compromiso } \\
\text { ético }\end{array}$ & $\begin{array}{l}\text { 17. Capacidad de } \\
\text { trabajo en equipo }\end{array}$ & $\begin{array}{c}\text { 4. Conocimientos } \\
\text { sobre el área de } \\
\text { estudio y la profesión }\end{array}$ & $\begin{array}{l}\text { 19. Capacidad para } \\
\text { motivar y conducir } \\
\text { hacia metas comunes }\end{array}$ \\
\hline 4 & $\begin{array}{l}\text { 27. Compromiso } \\
\text { con la calidad }\end{array}$ & $\begin{array}{l}\text { 18. Habilidades } \\
\text { interpersonales }\end{array}$ & $\begin{array}{l}\text { 17. Capacidad de } \\
\text { trabajo en equipo }\end{array}$ & $\begin{array}{l}\text { 16. Capacidad para } \\
\text { tomar decisiones }\end{array}$ \\
\hline 5 & $\begin{array}{c}5 . \\
\text { Responsabilidad } \\
\text { social y } \\
\text { compromiso } \\
\text { ciudadano }\end{array}$ & $\begin{array}{c}\text { 2. Capacidad } \\
\text { para aplicar los } \\
\text { conocimientos en la } \\
\text { práctica }\end{array}$ & $\begin{array}{c}\text { 10. Capacidad } \\
\text { para aprender } \\
\text { y actualizarse } \\
\text { permanentemente }\end{array}$ & $\begin{array}{l}\text { 26. Compromiso } \\
\text { ético }\end{array}$ \\
\hline
\end{tabular}

Fuente: elaboración propia

\section{Ranking de competencias genéricas} y específicas. El análisis estadístico en función de las medidas de centramiento permitió establecer las cinco competencias genéricas y específicas más importantes, en mayor grado de desarrollo, desde la percepción de los diferentes grupos de consulta (académicos, egresados, empleadores y estudiantes). Estos resultados se presentan en las tablas 5 y 6 .
Considerar el grado de importancia es uno de los factores más influyentes en la construcción de un ranking de competencias pues representa en esencia el interés de los grupos analizados. A partir de los resultados de la tabla 5 y 6 , se realizó un análisis de frecuencia que permitió establecer un ranking de competencias genéricas y específicas en función del grado de importancia. En las tablas 7 y 8 se presentan los resultados del ranking. 
Tabla 6

Resultados del análisis para las competencias específicas

\begin{tabular}{|c|c|c|c|c|}
\hline $\begin{array}{l}\text { Ranking de } \\
\text { competencias } \\
\text { específicas: } \\
\text { importancia }\end{array}$ & Estudiantes & Académicos & Graduados & Empresarios \\
\hline 1 & $\begin{array}{l}\text { 12. Ejercer el } \\
\text { liderazgo para } \\
\text { lograr y conseguir } \\
\text { metas en la } \\
\text { organización }\end{array}$ & $\begin{array}{l}\text { 1. Desarrollar } \\
\text { un planeamiento } \\
\text { estratégico, táctico y } \\
\text { operativo }\end{array}$ & $\begin{array}{l}\text { 21. Administrar los } \\
\text { recursos humanos, } \\
\text { físicos, financieros } \\
\text { y otros de la } \\
\text { organización }\end{array}$ & $\begin{array}{l}\text { 12. Ejercer el } \\
\text { liderazgo para } \\
\text { lograr y conseguir } \\
\text { metas en la } \\
\text { organización }\end{array}$ \\
\hline 2 & $\begin{array}{l}\text { 13. Administrar } \\
\text { y desarrollar el } \\
\text { talento humano en } \\
\text { la organización }\end{array}$ & $\begin{array}{l}\text { 12. Ejercer el } \\
\text { liderazgo para } \\
\text { lograr y conseguir } \\
\text { metas en la } \\
\text { organización }\end{array}$ & $\begin{array}{l}\text { 12. Ejercer el } \\
\text { liderazgo para } \\
\text { lograr y conseguir } \\
\text { metas en la } \\
\text { organización }\end{array}$ & $\begin{array}{l}\text { 17. Utilizar las } \\
\text { tecnologías de } \\
\text { información y } \\
\text { comunicación en la } \\
\text { gestión }\end{array}$ \\
\hline 3 & $\begin{array}{l}21 . \text { Administrar los } \\
\text { recursos humanos, } \\
\text { físicos, financieros } \\
\text { y otros de la } \\
\text { organización }\end{array}$ & $\begin{array}{l}21 . \text { Administrar los } \\
\text { recursos humanos, } \\
\text { físicos, financieros } \\
\text { y otros de la } \\
\text { organización }\end{array}$ & $\begin{array}{l}\text { 9. Interpretar la } \\
\text { información contable } \\
\text { y la información } \\
\text { financiera para la } \\
\text { toma de decisiones } \\
\text { gerenciales }\end{array}$ & $\begin{array}{l}\text { 1. Desarrollar } \\
\text { un planeamiento } \\
\text { estratégico, táctico } \\
\text { y operativo }\end{array}$ \\
\hline 4 & $\begin{array}{l}\text { 15. Mejorar e } \\
\text { innovar los procesos } \\
\text { administrativos }\end{array}$ & $\begin{array}{l}\text { 9. Interpretar la } \\
\text { información contable } \\
\text { y la información } \\
\text { financiera para la } \\
\text { toma de decisiones } \\
\text { gerenciales }\end{array}$ & $\begin{array}{l}\text { 11. Tomar decisiones } \\
\text { de inversión, } \\
\text { financiamiento y } \\
\text { gestión de recursos } \\
\text { financieros en la } \\
\text { empresa }\end{array}$ & $\begin{array}{l}\text { 6. Identificar las } \\
\text { interrelaciones } \\
\text { funcionales de la } \\
\text { organización }\end{array}$ \\
\hline 5 & $\begin{array}{l}\text { 11. Tomar } \\
\text { decisiones } \\
\text { de inversión, } \\
\text { financiamiento y } \\
\text { gestión de recursos } \\
\text { financieros en la } \\
\text { empresa }\end{array}$ & $\begin{array}{l}\text { 11. Tomar decisiones } \\
\text { de inversión, } \\
\text { financiamiento y } \\
\text { gestión de recursos } \\
\text { financieros en la } \\
\text { empresa }\end{array}$ & $\begin{array}{l}\text { 5. Desarrollar, } \\
\text { implementar } \\
\text { y gestionar } \\
\text { sistemas de control } \\
\text { administrativo }\end{array}$ & $\begin{array}{l}\text { 15. Mejorar } \\
\text { e innovar } \\
\text { los procesos } \\
\text { administrativos }\end{array}$ \\
\hline
\end{tabular}




\begin{tabular}{|c|c|c|c|c|}
\hline $\begin{array}{l}\text { Ranking de } \\
\text { competencias } \\
\text { específicas: } \\
\text { grado de } \\
\text { desarrollo }\end{array}$ & Estudiantes & Académicos & Graduados & Empresarios \\
\hline 1 & $\begin{array}{l}\text { 13. Administrar } \\
\text { y desarrollar el } \\
\text { talento humano en } \\
\text { la organización }\end{array}$ & $\begin{array}{l}21 . \text { Administrar los } \\
\text { recursos humanos, } \\
\text { físicos, financieros } \\
\text { y otros de la } \\
\text { organización }\end{array}$ & $\begin{array}{l}\text { 13. Administrar } \\
\text { y desarrollar el } \\
\text { talento humano en } \\
\text { la organización }\end{array}$ & $\begin{array}{l}\text { 12. Ejercer el } \\
\text { liderazgo para el } \\
\text { logro y consecución } \\
\text { de metas en la } \\
\text { organización }\end{array}$ \\
\hline 2 & $\begin{array}{l}\text { 12. Ejercer el } \\
\text { liderazgo para el } \\
\text { logro y consecución } \\
\text { de metas en la } \\
\text { organización }\end{array}$ & $\begin{array}{l}\text { 1. Desarrollar } \\
\text { un planeamiento } \\
\text { estratégico, táctico y } \\
\text { operativo }\end{array}$ & $\begin{array}{l}\text { 21. Administrar los } \\
\text { recursos humanos, } \\
\text { físicos, financieros } \\
\text { y otros de la } \\
\text { organización }\end{array}$ & $\begin{array}{l}\text { 17. Utilizar las } \\
\text { tecnologías de } \\
\text { información y } \\
\text { comunicación en la } \\
\text { gestión }\end{array}$ \\
\hline 3 & $\begin{array}{l}\text { 20. Formular planes } \\
\text { de marketing }\end{array}$ & $\begin{array}{l}\text { 11. Tomar decisiones } \\
\text { de inversión, } \\
\text { financiamiento y } \\
\text { gestión de recursos } \\
\text { financieros en la } \\
\text { empresa }\end{array}$ & $\begin{array}{l}\text { 9. Interpretar la } \\
\text { información contable } \\
\text { y la información } \\
\text { financiera para la } \\
\text { toma de decisiones } \\
\text { gerenciales }\end{array}$ & $\begin{array}{l}\text { 6. Identificar las } \\
\text { interrelaciones } \\
\text { funcionales de la } \\
\text { organización }\end{array}$ \\
\hline 4 & $\begin{array}{l}\text { 21. Administrar los } \\
\text { recursos humanos, } \\
\text { físicos, financieros } \\
\text { y otros de la } \\
\text { organización }\end{array}$ & $\begin{array}{l}\text { 5. Desarrollar, } \\
\text { implementar } \\
\text { y gestionar } \\
\text { sistemas de control } \\
\text { administrativo }\end{array}$ & $\begin{array}{l}\text { 12. Ejercer el } \\
\text { liderazgo para } \\
\text { lograr y conseguir } \\
\text { metas en la } \\
\text { organización }\end{array}$ & $\begin{array}{l}\text { 15. Mejorar } \\
\text { e innovar } \\
\text { los procesos } \\
\text { administrativos }\end{array}$ \\
\hline 5 & $\begin{array}{l}\text { 17. Utilizar las } \\
\text { tecnologías de } \\
\text { información y } \\
\text { comunicación en la } \\
\text { gestión }\end{array}$ & $\begin{array}{l}\text { 9. Interpretar la } \\
\text { información contable } \\
\text { y la información } \\
\text { financiera para la } \\
\text { toma de decisiones } \\
\text { gerenciales }\end{array}$ & $\begin{array}{l}\text { 15. Mejorar e } \\
\text { innovar los procesos } \\
\text { administrativos }\end{array}$ & $\begin{array}{l}\text { 2. Identificar } \\
\text { y administrar } \\
\text { los riesgos de } \\
\text { negocios de las } \\
\text { organizaciones }\end{array}$ \\
\hline
\end{tabular}

Fuente: elaboración propia.

Establecer un ranking de competencias genéricas y específicas a partir de la percepción de los grupos estudiados en la in- vestigación se convierte en la base para la construcción de un perfil del administrador acorde con las necesidades del entorno, asi- 
Tabla 7

Ranking de las cinco competencias genéricas más importantes.

\begin{tabular}{|c|c|c|c|c|c|c|c|c|c|c|c|}
\hline $\begin{array}{l}\text { Competencias } \\
\text { genéricas }\end{array}$ & 26 & 17 & 27 & 2 & 16 & 28 & 15 & 1 & 10 & 6 & 4 \\
\hline Repeticiones & 4 & 3 & 3 & 2 & 2 & 1 & 1 & 1 & 1 & 1 & 1 \\
\hline \multicolumn{12}{|c|}{ Ranking de las cinco competencias genéricas más importantes. } \\
\hline 26 & \multicolumn{11}{|c|}{ Compromiso ético } \\
\hline 17 & \multicolumn{11}{|c|}{ Capacidad de trabajo en equipo } \\
\hline 27 & \multicolumn{11}{|c|}{ Compromiso con la calidad } \\
\hline 2 & \multicolumn{11}{|c|}{ Capacidad para aplicar los conocimientos en la práctica } \\
\hline 16 & \multicolumn{11}{|c|}{ Capacidad para tomar decisiones } \\
\hline
\end{tabular}

Fuente: elaboración propia.

Tabla 8

Ranking de las cinco competencias específicas más importantes.

\begin{tabular}{|c|c|c|c|c|c|c|c|c|c|c|c|}
\hline $\begin{array}{l}\text { Competencias } \\
\text { específicas }\end{array}$ & 12 & 21 & 11 & 1 & 9 & 15 & 13 & 17 & 6 & 5 & - \\
\hline Repeticiones & 4 & 3 & 3 & 2 & 2 & 2 & 1 & 1 & 1 & 1 & . \\
\hline \multicolumn{11}{|c|}{ Ranking de las cinco competencias específicas más importantes. } & \\
\hline 12 & \multicolumn{10}{|c|}{ Ejercer el liderazgo para lograr y conseguir metas en la organización } & \\
\hline 21 & \multicolumn{10}{|c|}{ Administrar los recursos humanos, físicos, financieros y otros de la organización } & \\
\hline 11 & \multicolumn{11}{|c|}{$\begin{array}{l}\text { Tomar decisiones de inversión, financiamiento y gestión de recursos financieros en la } \\
\text { empresa }\end{array}$} \\
\hline 1 & \multicolumn{10}{|c|}{ Desarrollar un planeamiento estratégico, táctico y operativo } & \\
\hline 9 & \multicolumn{10}{|c|}{$\begin{array}{l}\text { Interpretar la información contable y la información financiera para la toma de } \\
\text { decisiones gerenciales }\end{array}$} & \\
\hline
\end{tabular}

Fuente: elaboración propia.

mismo, refleja de alguna u otra forma las competencias a las que requiere otorgárseles mayor relevancia en la formación de estudiantes en el programa de Administración de Empresas de la Universidad de la Costa. 
Se resalta de la construcción de ranking de competencias genéricas y específicas la participación de empresarios y egresados pues, según el Centro Interuniversitario de Desarrollo y la Universidad del Norte (2005), no es habitual en las instituciones formadoras la participación de empleadores y egresados en el proceso de diseño curricular, por lo general, el currículo es establecido sobre la base del saber científico y erudito de sus propios académicos. No obstante, cabe anotar que en la actualidad muchas instituciones de educación superior vinculan a empresarios y egresados para la elaboración de los diseños curriculares pero no para la identificación de competencias.

Percepción de los grupos de interés del estudio con relación a los reportados en el Proyecto Tuning-Colombia. Los resultados de las comparaciones entre la importancia y el grado de realización del país, con respecto a lo encontrado en el programa de Administración de Empresas de la Universidad de la Costa se presentan en las tablas 9, 10,11y 12 .

Tabla 9

Pruebas de comparación de las competencias de los estudiantes de la Universidad de la Costa con el país.

\begin{tabular}{|c|c|c|c|c|c|c|c|c|}
\hline & \multicolumn{5}{|c|}{ Diferencias relacionadas } & \multirow{3}{*}{$\mathrm{t}$} & \multirow{3}{*}{ gl } & \multirow{3}{*}{$\begin{array}{c}\text { Sig. } \\
\text { (bilateral) }\end{array}$} \\
\hline & \multirow[t]{2}{*}{ Media } & \multirow[t]{2}{*}{$\begin{array}{c}\text { Desviación } \\
\text { típ. }\end{array}$} & \multirow{2}{*}{$\begin{array}{c}\text { Error } \\
\text { típ. } \\
\text { de la } \\
\text { media }\end{array}$} & \multicolumn{2}{|c|}{$\begin{array}{c}\text { 95\% Intervalo de } \\
\text { confianza para la } \\
\text { diferencia } \\
\end{array}$} & & & \\
\hline & & & & Inferior & Superior & & & \\
\hline $\begin{array}{l}\text { Importancia de las } \\
\text { competencias específicas en } \\
\text { estudiantes de la CUC - País }\end{array}$ &,- 0077907 & ,0773338 & ,0172924 &,- 0439840 &, 0284027 &,- 451 & 19 &, 657 \\
\hline $\begin{array}{l}\text { Nivel de realización de las } \\
\text { competencias específicas en } \\
\text { estudiantes de la CUC - País }\end{array}$ & ,1359932 & 0915100 & ,0204623 & ,0931652 &, 1788212 & 6,646 & 19 &, 000 \\
\hline $\begin{array}{l}\text { Importancia de las } \\
\text { competencias genéricas en } \\
\text { estudiantes de la CUC - País }\end{array}$ & ,0120108 &, 1164514 &, 0224111 &,- 0340558 &, 0580774 &, 536 & 26 &, 597 \\
\hline $\begin{array}{l}\text { Nivel de realización de las } \\
\text { competencias genéricas en } \\
\text { estudiantes de la CUC - País }\end{array}$ & ,2686672 &, 1658217 & ,0319124 & ,2030703 &, 3342641 & 8,419 & 26 &, 000 \\
\hline
\end{tabular}

Fuente: elaboración propia. 
Los resultados de la tabla 9 permiten determinar que cuando se compara la importancia de las competencias específicas y las genéricas de los estudiantes con respecto a lo que ocurre a nivel del país, no se encuentra diferencia significativa. Pero, con respecto a la realización, sí existe una diferencia significativa. Esto indica que los estudiantes de la universidad tienen percepciones similares a los estudiantes del ámbito nacional en lo referente a las competencias genéricas y específicas. Ahora, es claro que cuando la comparación se hace con los grados de realización a nivel del país las percepciones difieren.

De acuerdo con los resultados de la tabla 10, se encuentra una diferencia significativa con respecto a la percepción de los académicos sobre la importancia de las competencias específicas y genéricas, en contraste con lo que ocurre a nivel del país; aspecto que es igual en los grados de realización. Esto indica que los académicos de

Tabla 10

Pruebas de comparación de las percepciones de las competencias de los académicos de la Universidad de la Costa con el país.

\begin{tabular}{|c|c|c|c|c|c|c|c|c|}
\hline & \multicolumn{5}{|c|}{ Diferencias relacionadas } & \multirow{3}{*}{$\mathrm{t}$} & \multirow{3}{*}{ gl } & \multirow{3}{*}{$\begin{array}{c}\text { Sig. } \\
\text { (bilateral) }\end{array}$} \\
\hline & \multirow[t]{2}{*}{ Media } & \multirow[t]{2}{*}{$\begin{array}{c}\text { Desviación } \\
\text { típ. }\end{array}$} & \multirow{2}{*}{$\begin{array}{c}\text { Error } \\
\text { típ. } \\
\text { de la } \\
\text { media }\end{array}$} & \multicolumn{2}{|c|}{$\begin{array}{c}\text { 95\% Intervalo de } \\
\text { confianza para la } \\
\text { diferencia }\end{array}$} & & & \\
\hline & & & & Inferior & Superior & & & \\
\hline $\begin{array}{c}\text { Importancia de las } \\
\text { competencias específicas en } \\
\text { académicos de la CUC - País }\end{array}$ & ,0398824 & ,0549626 & ,0122900 & ,0141590 &, 0656057 & 3,245 & 19 &, 004 \\
\hline $\begin{array}{l}\text { Nivel de realización de las } \\
\text { competencias específicas en } \\
\text { académicos de la CUC - País }\end{array}$ &, 3252647 & ,1032129 & ,0230791 & ,2769596 & ,3735698 & 14,093 & 19 &, 000 \\
\hline $\begin{array}{l}\text { Importancia de las } \\
\text { competencias genéricas en } \\
\text { académicos de la CUC - País }\end{array}$ & 1424183 &, 1281741 & ,0246671 & 0917143 & ,1931223 & 5,774 & 26 &, 000 \\
\hline $\begin{array}{l}\text { Nivel de realización de las } \\
\text { competencias genéricas en } \\
\text { académicos de la CUC - País }\end{array}$ & ,3316122 & ,1992363 & 0383431 & ,2527969 & ,4104275 & 8,649 & 26 &, 000 \\
\hline
\end{tabular}

Fuente: elaboración propia. 
la universidad tienen percepciones distintas con respecto a los académicos del ámbito nacional en lo referente a las competencias genéricas y específicas en sus dimensiones de importancia y niveles de realización. Se considera que la principal causa es el contexto.

En la tabla 11, los resultados permiten afirmar que la percepción de los graduados con relación a la importancia de las compe- tencias específicas y genéricas, en comparación con lo que ocurre a nivel del país, no se encuentra una diferencia significativa; aspecto que es igual en los grados de realización. Esto indica que los graduados de la universidad tienen percepciones iguales a los graduados con respecto al ámbito nacional en lo referente a las competencias genéricas y específicas en sus dimensiones de importancia y grado de realización. Es

Tabla 11

Pruebas de comparación de las percepciones de las competencias de los graduados de la Universidad de la Costa con el país.

\begin{tabular}{|c|c|c|c|c|c|c|c|c|}
\hline & \multicolumn{5}{|c|}{ Diferencias relacionadas } & \multirow{3}{*}{$\mathrm{t}$} & \multirow{3}{*}{$\mathrm{gl}$} & \multirow{3}{*}{$\begin{array}{l}\text { Sig. } \\
\text { (bilateral) }\end{array}$} \\
\hline & \multirow[t]{2}{*}{ Media } & \multirow{2}{*}{$\begin{array}{l}\text { Desviación } \\
\text { típ. }\end{array}$} & \multirow{2}{*}{$\begin{array}{l}\text { Error } \\
\text { típ. de la } \\
\text { media }\end{array}$} & \multicolumn{2}{|c|}{$\begin{array}{c}\text { 95\% Intervalo de } \\
\text { confianza para la } \\
\text { diferencia }\end{array}$} & & & \\
\hline & & & & Inferior & Superior & & & \\
\hline $\begin{array}{l}\text { Importancia de las } \\
\text { competencias específicas en } \\
\text { graduados de la CUC - País }\end{array}$ &,- 0136504 & 0951837 & ,0212837 &,- 0581977 & ,0308970 &,- 641 & 19 &, 529 \\
\hline $\begin{array}{l}\text { Nivel de realización de las } \\
\text { competencias específicas en } \\
\text { graduados de la CUC - País }\end{array}$ &,- 0418191 & ,1010188 & 0225885 &,- 0890973 &, 0054592 & $-1,851$ & 19 &, 080 \\
\hline $\begin{array}{l}\text { Importancia de las } \\
\text { competencias genéricas en } \\
\text { graduados de la CUC - País }\end{array}$ & ,0270277 & ,1205907 & ,0232077 &,- 0206764 & ,0747318 & 1,165 & 26 &, 255 \\
\hline $\begin{array}{l}\text { Nivel de realización de las } \\
\text { competencias genéricas en } \\
\text { graduados de la CUC - País }\end{array}$ & 0391853 & ,1103119 & ,0212295 &,- 0044527 &, 0828232 & 1,846 & 26 &, 076 \\
\hline
\end{tabular}

Fuente: elaboración propia. 
Tabla 12

Pruebas de comparación de las percepciones de las competencias de los empleadores con el país.

\begin{tabular}{|c|c|c|c|c|c|c|c|c|}
\hline & \multicolumn{5}{|c|}{ Diferencias relacionadas } & \multirow{3}{*}{$\mathrm{t}$} & \multirow{3}{*}{$\mathrm{gl}$} & \multirow{3}{*}{$\begin{array}{c}\text { Sig. } \\
\text { (bilateral) }\end{array}$} \\
\hline & \multirow[t]{2}{*}{ Media } & \multirow[t]{2}{*}{$\begin{array}{c}\text { Desviación } \\
\text { típ. }\end{array}$} & \multirow{2}{*}{$\begin{array}{c}\text { Error } \\
\text { típ. } \\
\text { de la } \\
\text { media }\end{array}$} & \multicolumn{2}{|c|}{$\begin{array}{c}\text { 95\% Intervalo de } \\
\text { confianza para la } \\
\text { diferencia }\end{array}$} & & & \\
\hline & & & & Inferior & Superior & & & \\
\hline $\begin{array}{l}\text { Importancia de } \\
\text { las competencias } \\
\text { específicas en } \\
\text { empleadores - País }\end{array}$ &,- 0453478 & ,1600475 & ,0357877 &,- 1202524 & ,0295567 & $-1,267$ & 19 &, 220 \\
\hline $\begin{array}{l}\text { Nivel de realización } \\
\text { de las competencias } \\
\text { específicas en } \\
\text { empleadores - País }\end{array}$ & ,3548043 &, 2542950 & 0568621 & ,2357906 & ,4738181 & 6,240 & 19 &, 000 \\
\hline $\begin{array}{l}\text { Importancia de } \\
\text { las competencias } \\
\text { genéricas en } \\
\text { empleadores - País }\end{array}$ &,- 0289372 &, 1400244 & 0269477 &,- 0843290 & ,0264546 & $-1,074$ & 26 &, 293 \\
\hline $\begin{array}{l}\text { nivel de realización } \\
\text { de las competencias } \\
\text { genéricas en } \\
\text { empleadores - País }\end{array}$ &, 5321900 & ,2588878 & 0498230 & ,4297774 & ,6346026 & 10,682 & 26 &, 000 \\
\hline
\end{tabular}

Fuente: Elaboración propia

posible que esto se presente debido a que los graduados muchas veces trabajan en diferentes lugares del país, de allí que exista una tendencia a considerar que las competencias más importantes y que han desarrollado sean las mismas competencias que se presentan a nivel país.

Los resultados de la tabla 12 permiten afirmar que cuando se compara la impor- tancia de las competencias específicas con las genéricas de los empleadores con respecto a lo que ocurre a nivel del país no se encuentra una diferencia significativa. Pero, en los niveles de realización, sí existe una diferencia significativa. Esto indica que los empleadores de la universidad tienen percepciones parecidas a los estudiantes en lo referente con las competencias genéricas y específicas. 


\section{Conclusiones}

Identificar el perfil del administrador de empresas egresado de la Universidad de la Costa, Colombia, para contrastarlo con las competencias establecidas en el proyecto Tuning-Colombia permitió determinar la importancia de afianzar el referido proceso desde una visión centrada en su sistematicidad para la definición de competencias genéricas y específicas, las cuales ameritan ser concebidas en el marco de la formación integral del individuo. Como parte del proceso de contrastación, se destacan las competencias generales vistas desde el compromiso ético, así como con la calidad, el trabajo en equipo, el dominio y efectividad en la aplicación de conocimientos en la práctica y, por ende, la asertividad en el proceso de toma de decisiones responden a las cinco competencias genéricas de mayor interés para el grupo de análisis.

Con respecto a la contrastación de las competencias específicas, las de mayor interés para el grupo de análisis responden a ejercer el liderazgo para el logro y consecución de metas en la organización; administrar los recursos humanos, físicos, financieros y otros de la organización; tomar decisiones de inversión, financiamiento y gestión de recursos financieros en la empresa; desarrollar un plantea- miento estratégico, táctico y operativo; e interpretar la información contable y la información financiera para la toma de decisiones gerenciales. El compromiso ético, relacionado con las competencias genéricas, así como la capacidad de ejercer el liderazgo para el logro y consecución de metas en la organización, referidas a las competencias específicas, se conciben como las más destacadas, tanto en el grado de importancia como de desarrollo para el grupo de análisis.

Para las competencias específicas, orientadas hacia la administración de los recursos humanos, físicos y financieros, así como a la administración y desarrollo del talento humano son consideradas por los grupos de interés como las competencias con un alto grado de desarrollo. No obstante, se resalta que para los empresarios la de mayor relevancia es la de ejercer el liderazgo para el logro y consecución de metas en la organización.

En este orden de ideas, el profesional del programa de Administración de Empresas de la Universidad de la Costa se caracteriza por el desarrollo de competencias que le permiten la interacción como emprendedor e innovador, capaz de promover la toma decisiones y liderar iniciativas que respalden el logro y la consecución de metas en una organización de manera eficien- 
te, lo cual responde al emprendimiento de procesos educativos basado en competencias, actualmente concebida como la principal tendencia de los modelos asumidos en las instituciones de educación superior del contexto colombiano.

En tal sentido, afianzar el desarrollo del perfil del administrador basados en competencias requiere de la sistematicidad de los procesos formativos y continuidad, donde, de forma integrada, políticas educativas, infraestructuras y relaciones interinstitucionales, tanto para el profesorado como para los estudiantes, conlleven a elevar los estándares de un nuevo perfil centrado en la innovación y el emprendimiento.

En líneas generales, la definición del perfil no sólo responde a las características o competencias asociadas a un profesional. Éste necesariamente responde al diseño curricular y, por ende, al desarrollo de estrategias pedagógicas que garanticen la concreción de competencias. Aunado a esto, Daza, Charris y Viloria (2015), señalan que se puede generar el camino para el éxito empresarial desde la academia, traducido en la elaboración de planes de estudio homologables entre instituciones de educación superior regionales, nacionales e internacionales.

\section{Referencias}

Aguilar, J. (Junio, 2011). Las competencias de los administradores en Colombia y del sur occidente colombiano a la luz del proyecto Tuning América Latina. En OEA, XII encuentro internacional Virtual Educa. México D.F., México.

ASCOLFA. (2010). Las competencias de los administradores en Colombia a la luz del proyecto Tuning América Latina. Bogotá: Guía publicidad y asociados LTDA.

Beneitone, P., Esquetini, C., González, J., Maletá, M., Siufi, G. y Wagenaar, R. (2007). Reflexiones y perspectivas de la educación superior en América Latina. Informe Final -Proyecto Tuning-América Latina 2004-2007. Bilbao: Universidad de Deusto y Universidad de Groninguen.

Bravo, N. (2007). Competencias proyecto Tuning-Europa, Tuning.-América Latina. Recuperado de http://www.cca. org.mx/profesores/cursos/hmfbcp_ut/ $\mathrm{pdfs} / \mathrm{m} 1 /$ competencias_proyectotuning.pdf 
Centro Interuniversitario de Desarrollo - CINDA y la Universidad del Norte. (2005). Currículo universitario basado en competencias. Recuperado de http://www.cinda.cl/download/libros/ Curr\%C3\%ADculo\%20Universitario\%20Basado\%20en\%20Competencias.pdf

Daza, A., Charris, A., y Viloria, J. (2015). Importancia de competencias específicas de los administradores de la Región Caribe colombiana. Dimensión Empresarial, 13(2): 275 - 292. DOI: 10.15665/rde.v13i2.466

Organización para la Cooperación y el Desarrollo Económico -OCDE- y Banco Mundial. (2012). Evaluaciones de políticas nacionales de educación. La educación superior en Colombia. Recuperado de https://www.oecd.org/edu/ skills-beyond-school/Evaluaciones\%20 de\%20pol\%C3\%ADticas\%20nacionales\%20de\%20Educaci\%C3\%B3n\%20 -\%20La\%20Educaci\%C3\%B3n\%20superior\%20en\%20Colombia.pdf
UNESCO. (2009). Conferencia mundial sobre educación superior. Las nuevas dinámicas de la educación superior y de la investigación para el cambio social y el desarrollo. París: Ediciones UNESCO.

Universidad de Deusto - Universidad de Groningen. (2007). Tuning Educational Structures in Europe. Informe Final del Proyecto Piloto: Fase II. Recuperado de http://www.deusto-publicaciones.es/ deusto/pdfs/tuning/tuning04.pdf

Universidad de Deusto. (2014). Meta-perfiles y perfiles: Una nueva aproximación para las titulaciones en América Latina. Recuperado de www.tuningal.org/ es/publicaciones/doc_download/122-meta-perfiles-y-perfiles-una-nueva-aproximacion-para-las-titulaciones-en-america-latina-espanol

Valero, G. (2011). Las competencias de los administradores en Colombia a la luz del proyecto Tuning - caso capítulo oriente. Puente Revista Cientifica, 5(1). Recuperado de http://puente.upbbga. edu.co/index.php/revistapuente/article/ view/33/33 